\title{
Long-term Use of Aspirin and Age-related Macular Degeneration
}

\author{
William G Christen, $\mathrm{SCD}^{1}$ and Emily $\mathrm{Y}$ Chew, $\mathrm{MD}^{2}$ \\ 1. Associate Professor, Division of Preventive Medicine, Department of Medicine, Brigham and Women's Hospital and Harvard Medical School, Boston, Massachusetts, US, \\ 2. Deputy Director, Division of Epidemiology and Clinical Applications, National Eye Institute, National Institutes of Health, Bethesda, Maryland, US
}

\begin{abstract}
Recent findings from observational epidemiologic studies have raised concern about a possible adverse effect of regular aspirin use in agerelated macular degeneration (AMD), and in particular neovascular AMD, which is the leading cause of severe irreversible blindness in the US. In this report, we consider these findings in light of the relative strengths and limitations of observational studies and randomized trials. While the findings are important and warrant further investigation, the inherent limitations of observation studies, most notably uncontrolled confounding, preclude an interpretation of causality. Alternatively, the most reliable evidence with which to evaluate the effects of regular aspirin use in AMD will derive from well-designed randomized trials of sufficient size and duration.
\end{abstract}

\section{Keywords}

Aspirin, age-related macular degeneration, observational studies, randomized trials

Disclosure: The authors have no conflicts of interest to declare.

Received: July 17, 2013 Accepted: August 12, 2013 Citation: US Ophthalmic Review, 2013;6(2):144-7 DOI: 10.17925/USOR.2013.06.02.144

Correspondence: William G Christen, 900 Commonwealth Avenue, 3rd Floor, Boston, Massachusetts, 02215-1204. E: wchristen@rics.bwh.harvard.edu

\section{Aspirin in General Use}

Aspirin is used by many for temporary pain relief, fever reduction, rheumatologic diseases, and, where indicated, for cardioprotection. ${ }^{1}$ Accumulating evidence also suggests a possible beneficial role for aspirin in cancer prevention. ${ }^{2}$ However, aspirin has also been associated with significant adverse events such as cerebral and gastro-intestinal bleeding. Recent findings from several observational studies have raised concern that regular aspirin use may also be associated with increased risks for some forms of age-related macular degeneration (AMD), the leading cause of irreversible blindness in older Americans. In this report, we consider these recent findings for AMD in light of the relative strengths and limitations of observational studies and randomized trials.

\section{Aspirin and Age-related Macular Degeneration Early Observational Evidence}

The earliest reports of a possible link between aspirin and AMD were small case reports and case series that were prompted out of concern for possible increased risk for retinal hemorrhage for those who use aspirin. ${ }^{3-8}$ However, these studies were limited by the absence of a comparison group and thus were impossible to interpret. Subsequent cross-sectional, 9,10 case-control, ${ }^{11-13}$ and prospective cohort studies ${ }^{14-17}$ provide little evidence of a link between aspirin use and AMD.

\section{Recent Observational Studies}

Results of three recent observational studies, including two prospective cohort studies, have again raised concern in the ophthalmologic community and beyond about a possible deleterious effect of aspirin use in AMD.

\section{European Eye Study}

de Jong and colleagues ${ }^{18}$ examined the associations between aspirin use and early and late AMD in a population-based, cross-sectional study of 4,691 men and women, 65 years of age and older, randomly sampled from population registries of seven European countries. Information on aspirin use and possible confounders for AMD was ascertained through a structured questionnaire administered by study field workers. Aspirin use was categorized according to reported frequency into four groups: never, monthly or less, at least once weekly (but not daily), and daily. AMD was centrally classified using digitized fundus images and a modified International Classification System.

Early AMD was present in 1,706 (36.4\%) participants who were further categorized into three groups of increasing severity of early AMD (grades 1 to 3). Late AMD was present in 157 (3.3\%) participants including 108 (2.3\%) with wet, late (neovascular) AMD. Eight hundred thirty-nine (17.9\%) participants reported using aspirin daily, 326 (6.9\%) at least once weekly (but not daily), 766 (16.3\%) monthly or less, and 2,760 (58.8\%) never. Daily aspirin users were older, less likely to be a current smoker, but more likely to be a former smoker, and more likely to report a history of cardiovascular disease (CVD), angina, and diabetes.

Daily aspirin users, compared with never users, had an approximately twofold increased odds of neovascular AMD (odds ratio [OR] 2.26, $95 \%$ confidence interval [Cl] 1.66-3.08) in analyses adjusted for potential confounders. The ORs for early AMD were: grade 1, 1.27 (95 \% Cl 1.09-1.48); grade 2, 1.40 (95 \% Cl 1.16-1.68), and grade 3, 0.95 (95\% Cl 0.61-1.46). 


\section{Beaver Dam Eye Study}

Klein and colleagues ${ }^{19}$ examined the association between regular aspirin use and incident AMD in a longitudinal, population-based study of men and women aged 43 to 86 years in Wisconsin. A total of 3,206 participants with information on aspirin use and AMD status at baseline and at followup were included in analyses. Examinations were performed every 5 years over a 20-year period. Participants were asked at baseline and at each follow-up exam about possible AMD risk factors including aspirin use. Regular aspirin use was defined as use at least twice a week for more than 3 months. The presence and severity of lesions associated with AMD was assessed through review of retinal photographs taken at each examination and graded using the Wisconsin Age-Related Maculopathy Grading System.

During a median duration of 15.9 years of follow-up, the investigators identified 512 incident cases of early AMD and 117 cases of late AMD, including 78 cases of neovascular AMD. The percentage of participants categorized as regular users of aspirin varied according to follow-up interval and AMD endpoint examined, but averaged between $42 \%$ and $45 \%$. Specific information on the ways in which regular users of aspirin differed from nonregular users was not presented; however, analyses of early AMD included adjustment for age, sex, education level, ever heavy drinking, smoking, and history of arthritis, whereas analyses of late AMD included adjustment for age, sex, education, heavy drinking history, and smoking.

Regular aspirin use 10 years prior to retinal examination was associated with an increased risk for late AMD (hazard ratio [HR], 1.63, $95 \% \mathrm{Cl} 1.01-2.63$ ). This increased risk was specific for neovascular AMD (HR 2.20, 95\% Cl 1.20-4.15); there was no significant association with pure geographic atrophy (HR 0.66, $95 \% \mathrm{Cl} 0.25-1.95)$. Regular aspirin use 5 years prior to retinal examination was not associated with late AMD. Nor was there any association between incident early AMD and aspirin use 5 years (HR 0.86, $95 \% \mathrm{Cl} 0.71-1.05$ ) or 10 years (HR 0.86, $95 \%$ Cl 0.65-1.13) prior to retinal examination.

\section{Blue Mountains Eye Study}

Liew and colleagues examined the prospective relationship between long-term, low-dose aspirin use and risk for AMD in an Australian population-based cohort of 2,389 adult men and women aged 49 years or older. Participants completed a questionnaire at baseline providing information on a range of risk factors for AMD. Use of aspirin and other medications was ascertained during a structured interview at baseline using a standard questionnaire. Regular use of aspirin was defined as a reported frequency of one or more times per week in the past year, and occasional use as less than once per week in the past year. AMD status was assessed through review of retinal photographs obtained at baseline and at 5-year intervals over a 15-year follow-up period, and graded using an international AMD classification system.

During the 15-year follow-up, 63 participants developed neovascular AMD. A total of 257 (10.8 \%) of the 2,389 participants were classified as regular users of aspirin. Regular users were markedly older, and were more likely to report a personal history of stroke, heart disease, diabetes, and hypertension.

Regular users of aspirin, compared with nonregular users (nonusers plus occasional users), had a two- to threefold increased risk for incident neovascular AMD (OR 2.37, $95 \%$ Cl 1.25-4.49) in analyses adjusted for age, sex, and smoking. The OR changed little after further adjustment for history of CVD, body mass index, and systolic blood pressure. Further adjustment for additional CVD risk factors including blood pressure, cholesterol level, diabetes, fish consumption, and inflammatory markers, reduced the OR and it was no longer significant (OR 2.05, $95 \% \mathrm{Cl}$ 0.96-4.40). There was no significant association with geographic atrophy or early AMD.

\section{Commentary}

All three recently reported observational studies share several strengths including a well-characterized, population-based study sample, information on a range of risk factors for $\mathrm{AMD}$, and the use of retinal photographs and a standardized classification scheme to assess AMD status. For the two prospective studies, ${ }^{19,20}$ information on AMD risk factors was collected prior to the diagnosis of AMD, which is an additional strength. All three studies report a similar two- to threefold increased risk for neovascular $\mathrm{AMD}$ in regular users of aspirin. As noted in the Blue Mountain Eye Study report, ${ }^{20}$ if these results reflect a true causal relationship, there are serious implications for the millions of people using aspirin therapy.

However, these observational studies have several important limitations that need to be considered. An inherent limitation of all observational studies is the potential for uncontrolled, or uncontrollable, confounding. In all three studies, participants who chose to use aspirin regularly differed from nonregular users in important respects. They were markedly older, had more diabetes and hypertension, and were more likely to report a personal history of CVD.

In assessing a possible causal connection with AMD, it seems particularly important to stress the magnitude of the difference in reported CVD (and CVD risk factors) between users and nonusers of aspirin in these studies. For example, in the European Eye Study, $38 \%$ of daily aspirin users reported a history of CVD compared with $9 \%$ of never users and $11 \%$ of weekly users. In the Blue Mountains Eye Study, $40 \%$ of regular aspirin users reported a history of heart disease compared with $11 \%$ of nonregular users (occasional and never users). Because aspirin therapy is commonly recommended for persons with CVD, these differences are not surprising. However, AMD and CVD are postulated to share similar underlying mechanisms and risk factors, ${ }^{21}$ and thus the potential for confounding, particularly confounding by indication, is great.

All three studies attempted to account for these differences between users and nonusers by including terms for these factors in analyses. However, such factors as CVD and associated risk factors are difficult to measure and fully adjust for in analysis, leaving ample room for residual confounding. Moreover, regular users of aspirin may differ from nonregular users in ways that were not measured or, indeed, yet recognized which, if predictive of AMD occurrence, would raise further concern about uncontrolled confounding.

Misclassification of aspirin use also seems a possibility. In the primary analyses for all three studies, aspirin exposure was based only on reported frequency of use, and participants were categorized into two to four broadly defined usage groups. Two studies collected no information on dosage ${ }^{18,20}$ while the third study collected dosage information for secondary analyses. ${ }^{19}$ 
Another concern is the small number of events. In the Blue Mountains Eye Study, a total of 63 incident cases of neovascular AMD were observed, including only 15 cases among regular users of aspirin. ${ }^{20}$ In Beaver Dam, there were 78 incident cases of neovascular AMD, which included only 30 cases among participants who reported regular use of aspirin 10 years prior to the diagnosis of AMD. In the European Eye Study, there were 108 prevalent cases of neovascular AMD confirmed. The small number of events results in imprecise and unstable estimates of association. These estimates are further weakened when attempting to control for multiple confounders, particularly when cases are dropped because of missing information for possible confounders.

In summary, while aspirin use may be causally associated with neovascular $A M D$, it is also possible that aspirin use is simply a marker for underlying factors or conditions (confounding by indication) more closely associated with the development or progression of AMD. Observational studies are unlikely to be able to distinguish between these and other possibilities.

\section{Randomized Trials}

On the other hand, most inherent limitations of observational studies are not applicable to randomized trials in which a standard dosage of aspirin is randomly assigned to study participants. If sufficiently large, randomized trials can provide the strongest and most reliable evidence on which to make a judgment of causality. Two such trials have been conducted: one in men ${ }^{22}$ and one in women. ${ }^{23}$

\section{Physician's Health Study I}

The Physicians' Health Study I (PHS I) was a randomized, double-masked, placebo-controlled trial designed to test low-dose aspirin (325 mg every other day) and beta-carotene in the primary prevention of CVD and cancer in 22,071 apparently healthy US male physicians, aged 40 to 84.22 AMD was examined as a secondary endpoint among 21,216 physicians who did not report AMD at baseline. Cases of AMD were defined as a participant report confirmed by medical record review.

During an average of 5 years of treatment and follow-up, a total of 117 cases of AMD were confirmed, including 57 cases with best-corrected visual acuity reduced to 20/30 or worse due to AMD (visually significant AMD). Most cases of visually significant AMD were characterized by some combination of drusen and retinal pigment epithelium (RPE) changes representing an early stage of disease development.

For the primary endpoint of visually significant AMD, there were 25 cases in the aspirin group and 32 in the placebo group (relative risk [RR] 0.78, $95 \% \mathrm{Cl} 0.46-1.32)$. For total AMD, there were 51 cases in the aspirin group and 66 in the placebo group (RR 0.77, $95 \% \mathrm{Cl} 0.54-1.11)$. There were too few cases of late (exudative) AMD $(n=12)$ for analysis.

\section{Women's Health Study}

The Women's Health Study I (WHS) was a randomized, double-masked, placebo-controlled trial designed to examine low-dose aspirin (100 mg every other day) and vitamin $\mathrm{E}$ in the primary prevention of CVD and cancer in 39,876 apparently healthy female health professionals aged 45 or older. ${ }^{23}$ AMD was examined as a secondary endpoint among 39,421 women who did not report AMD at baseline. Cases of AMD were defined as a participant report confirmed by medical record review.
During an average of 10 years of treatment and follow-up, 593 cases of AMD were confirmed, including 245 cases of visually significant AMD. As in PHS I, most cases of visually significant AMD were characterized by some combination of drusen and RPE changes. A total of 55 cases of advanced AMD (neovascular AMD plus geographic atrophy) were also identified.

For the primary endpoint of visually significant AMD, there were 111 cases in the aspirin group and 134 in the placebo group (RR 0.82, $95 \% \mathrm{Cl} 0.64-$ 1.06). For total $A M D$, there were 302 cases in the aspirin group and 291 in the placebo group (RR 1.03, $95 \% \mathrm{Cl}$ 0.88-1.21). For advanced AMD, there were 26 cases in the aspirin group and 29 in the placebo group (RR 0.90, $95 \% \mathrm{Cl}$ 0.53-1.52).

When data from the PHS I and WHS were combined in a stratified proportional hazards model (with study as the strata), results indicated a possible, but statistically nonsignificant, $18 \%$ reduced risk for visually significant AMD for those assigned to aspirin (RR 0.82, $95 \% \mathrm{Cl} 0.65-1.03$ ). ${ }^{23}$

Thus, these randomized trial findings from men and women without a reported diagnosis of AMD at baseline do not support a harmful effect of aspirin use in AMD occurrence and, instead, are most consistent with a small to moderate beneficial effect among aspirin users. It should be noted, however, that these trials comprised highly motivated health professionals and the findings may not be generalizeable to other populations. In addition, it was not possible in the trials to conduct study-related eye exams for all study participants. Instead, AMD cases were identified through participant self-reports and subsequently confirmed through medical record review. Thus, it seems likely that in the trials, some under-ascertainment of AMD occurred that would have reduced study power. However, such underascertainment is not associated with bias in randomized comparisons.

\section{Future Directions}

While recent observational data have raised concern about possible adverse effects of aspirin use in late-stage neovascular AMD, additional observational data are unlikely to clarify whether the association is one of cause and effect. Data from randomized trials of sufficient size and duration are required to provide the most reliable estimate of aspirin's effect in $\mathrm{AMD}$, and whether the effect differs in early- and late-stage disease. In terms of current clinical practice, the recent observational findings are insufficient to warrant any change from widespread aspirin use. In particular, the benefits of regular aspirin use in prevention of recurrent vascular events in those at high risk (secondary prevention) are well-established, ${ }^{24}$ and not likely to change with subsequent findings for AMD. For the large majority of persons at moderate risk for CVD, aspirin reduces the risk for first myocardial infarction; however, data on strokes and vascular death remain inconclusive. Moreover, the absolute risk is low and it is difficult to get reliable estimates of benefit to risk ratio in primary prevention in subjects at moderate risk. ${ }^{24} \mathrm{~A}$ better understanding of aspirin's effect in AMD would help to clarify the benefit to risk ratio for these individuals at moderate risk for CVD.

\section{Summary}

Recent findings from observational studies have raised concern in the ophthalmologic community and beyond about a possible increased risk for neovascular AMD in regular users of aspirin. However, the inherent limitations of observational studies preclude an interpretation 
of causality. Additional data from well-designed randomized trials are required to provide the most reliable evidence to evaluate the effects of regular aspirin use in early- and late-stage AMD. While such information is unlikely to alter current recommendations for persons at high risk for CVD, it should help clarify the benefit to risk ratio of regular aspirin use in the large majority of individuals at moderate risk for CVD.
1. US Food and Drug Administration (FDA) Aspirin: Questions and Answers. FDA website. Available at: http://www.fda. gov/drugs/resourcesforyou/consumers/questionsanswers/ ucm071879.htm (accessed June 10, 2013)

2. Rothwell PM, Price JF, Fowkes FG, et al., Short-term effects of daily aspirin on cancer incidence, mortality, and non-vascular death: analysis of the time course of risks and benefits in 51 randomised controlled trials, Lancet, 2012:379(9826):1602-12.

3. Feman SS, Bartlett RE, Roth AM, Foos RY, Intraocular Feman SS, Bartlett RE, Roth AM, FooS RY, Intraocular
hemorrhage and blindness associated with systemic anticoagulation, JAMA, 1972;220(10):1354-5

4. Bloome MA, Ruiz RS, Massive spontaneous subretinal hemorrhage, Am J Ophthalmol, 1978;86(5):630-37.

5. el Baba F, Jarrett WH, 2nd, Harbin TS, Jr., et al., Massive hemorrhage complicating age-related macular degeneration. Clinicopathologic correlation and role of anticoagulants, Ophthalmology, 1986:93(12):1581-92.

6. Lewis H, Sloan SH, Foos RY, Massive intraocular hemorrhage associated with anticoagulation and age-related macular degeneration, Graefes Arch Clin Exp Ophthalmol, 1988;226(1):59-64

7. Kingham JD, Chen MC, Levy MH, Macular hemorrhage in the aging eye: the effects of anticoagulants, $N$ Eng/ I Med, 1988:318(17):1126-7.

8. Kinshuck D, Stevenson L, Complications of NSAID therapy in patients with macular disease, Surv Ophthalmol, 1992;37(2):149-50.
9. Kiernan DF, Hariprasad SM, Rusu IM, et al., Epidemiology of the association between anticoagulants and intraocula hemorrhage in patients with neovascular age-related macular degeneration, Retina, 2010;30(10):1573-8

10. Hirvela $\mathrm{H}$, Luukinen $\mathrm{H}$, Laara $\mathrm{E}$, et al., Risk factors of agerelated maculopathy in a population 70 years of age or older, Ophthalmology, 1996;103(6):871-7.

11. Rudnicka AR, MacCallum PK, Whitelocke R, Meade TW, Circulating markers of arterial thrombosis and late-stage age-related macular degeneration: a case-control study, Eye (Lond), 2010;24(7):1199-1206.

12. Tilanus MA, Vaandrager W, Cuypers $\mathrm{MH}$, et al., Relationship between anticoagulant medication and massive intraocular hemorrhage in age-related macular degeneration, Graefes Arch Clin Exp Ophthalmol, 2000;238(6):482-5.

13. Risk factors associated with age-related macular degeneration. A case-control study in the age-related eye disease study: Age-Related Eye Disease Study Report Number 3, Ophthalmology, 2000;107(12):2224-32.

14. Klein ML, Macular degeneration: is aspirin a risk fo progressive disease? JAMA, 1991;266(16):2279.

15. Persistent and recurrent neovascularization after krypton laser photocoagulation for neovascular lesions of age-related macular degeneration. Macular Photocoagulation Study Group, Arch Ophthalmol, 1990;108(6):825-31.

16. Recurrent choroidal neovascularization after argon laser photocoagulation for neovascular maculopathy. Macular Photocoagulation Study Group, Arch Ophthalmol,
1986:104(4):503-12

17. Klein R, Klein BE, Jensen SC, et al., Medication use and the 5-year incidence of early age-related maculopathy: the Beaver Dam Eye Study, Arch Ophthalmol, 2001;119(9):1354-9.

18. de Jong PT, Chakravarthy U, Rahu M, et al., Associations between aspirin use and aging macula disorder: the European Eye Study, Ophthalmology, 2012;119(1):112-18.

19. Klein BE, Howard KP, Gangnon RE, et al., Long-term use of aspirin and age-related macular degeneration, JAMA, 2012;308(23):2469-78.

20. Liew G, Mitchell P, Wong TY, Rochtchina E, Wang JJ, The association of aspirin use with age-related macular degeneration, JAMA, 2013;173(4):258-64.

21. Snow KK, Seddon JM, Do age-related macular degeneration and cardiovascular disease share common antecedents? Ophthalmic Epidemiol, 1999:6(2):125-43.

22. Christen WG, Glynn RJ, Ajani UA, et al., Age-related maculopathy in a randomized trial of low-dose aspirin among US physicians, Arch Ophthalmol, 2001;119(8):1143-9.

23. Christen WG, Glynn RJ, Chew EY, Buring JE, Low-dos aspirin and medical record-confirmed age-related macular degeneration in a randomized trial of women Ophthalmology, 2009;116(12):2386-92.

24. Hennekens $\mathrm{CH}$, Dalen JE, Aspirin in the treatment and prevention of cardiovascular disease: past and current perspectives and future directions, Am J Med, 2013;126(5):373-8 